\title{
MedienPädagogik
}

Zeitschrift für Theorie und Praxis der Medienbildung

Themenheft Nr. 33: Medienpädagogik und Didaktik der Informatik.

Eine Momentaufnahme disziplinärer Bezüge und schulpraktischer Entwicklungen. Herausgegeben von Torsten Brinda, Ira Diethelm, Sven Kommer und Klaus Rummler

\section{Digitale Bildung: ein interdisziplinäres Verständnis zwischen Medienpädagogik und Informatik}

Andreas Dengel

\begin{abstract}
Zusammenfassung
Das Erfordernis digitaler Bildung schon im Kindesalter ist heutzutage unbestritten. Zahlreiche Initiativen und Forschungsprojekte beschäftigen sich mit der Etablierung Digitaler Bildung in weiterführenden Schulen, Hochschulen und berufsbegleitenden Fortbildungsmassnahmen. Hierbei wird allerdings nur selten ein definitorisches Verständnis Digitaler Bildung gegeben. Auch die Inhalts- und Kompetenzfrage in Deutschland gestaltet sich aktuell als Spannungsfeld zwischen medienpädagogischen und informatischen Inhalten. Dieses Paper versucht, ein interdisziplinäres Verständnis Digitaler Bildung zu generieren. Hierfür werden zunächst anhand des aktuellen Strategiepapiers «Bildung in der digitalen Welt» der Kultusministerkonferenz und deren Kritiken die Bedarfe Digitaler Bildung erörtert. Perspektiven aus der Medienpädagogik und der Informatik erläutern notwendige Inhaltsbereiche der Digitalen Bildung. Zusammen mit bereits bestehenden Begriffsdiskussionen wird versucht, ein gemeinsames Verständnis zu Digitaler Bildung zu schaffen.
\end{abstract}

Digital Literacy: an Interdisciplinary Understanding between Computer Science and Media Education

\begin{abstract}
As an essential part of today's early education, Digital Literacy is part of many research projects and initiatives in schools and universities. A uniform definition of the term «Digitale Bildung» currently does not exist in the German-speaking area. The topics covered by Digital Literacy seem to be between the conflicting priorities of Media Education and Computer Science. This paper tries to generate an integrative understanding of the term «Digitale Bildung» by merging the content of the strategy paper «Bildung in der digitalen Welt» from the german Kultusministerium and its critical reviews from different views. In the next step, short insights into the perspectives of researchers of the Media Education field of research and the Computer Science Community «Gesellschaft der Informatik» on Digital Literacy are complemented with existing definitions of «Digitale Bildung». Finally, an attempt to merge the discovered components of Digital Literacy to form an integrative understanding of «Digitale Bildung» is made.
\end{abstract}




\section{Digitale Bildung in Deutschland}

Nahezu allen Bildungsinitiativen der heutigen Zeit zum Thema «Digitale Bildung» ist eines gemein: Der Begriff selbst wird nie definiert (siehe hierzu bspw. «Bildung in der digitalen Welt»: Kultusministerium 2016; «Die richtige Bildung für die digitale Welt»: Digitaler Bildungspakt 2016a; «Digitale Bildung Neu Denken»: i-dbnd 2017).

Um jedoch Klarheit zum theoretischen Bezugsrahmen von Initiativen, Forschungsvorhaben oder Diskussionen zu schaffen, ist eine formale Begriffsdefinition unumgänglich. Diese Arbeit versucht, aus verschiedenen Perspektiven auf Digitale Bildung ein einheitliches Verständnis zu extrahieren: Zunächst wird die aktuell angestrebte Umsetzung Digitaler Bildung in der deutschen Bildungspolitik anhand des Strategiepapiers "Bildung in der digitalen Welt» betrachtet. Anschliessend werden die Perspektiven von Medienpädagogik und Informatik auf Digitale Bildung erörtert. Im Anschluss daran werden bereits bestehende Definitionen Digitaler Bildung selektiert, welche abschliessend mit den verschiedenen Perspektiven der Medienpädagogik und der Informatik sowie den Kompetenzbereichen der bestehenden Anwendungsgebiete verglichen werden. Natürlich erhebt diese Arbeit keinesfalls den Anspruch, den gesamten Diskurs zu Digitaler Bildung abzudecken. Es werden explorativ Sichtweisen und Definitionen aus möglichst verschiedenen Bereichen, auch mit unterschiedlichen Publikationsdaten, gewählt, um zunächst ein möglichst heterogenes Bild der Digitalen Bildung zu erschaffen. Hieraus wird abschliessend versucht, die richtungsgebenden Merkmale Digitaler Bildung in diesen verschiedenen Anwendungsbereichen zu extrahieren und ein interdisziplinäres Verständnis zwischen der Informatik und der Medienpädagogik für die Digitale Bildung zu generieren.

Die Kultusministerkonferenz (KMK) formulierte Dezember 2016 ihren neuen Beschluss «Bildung in der digitalen Welt» als Strategiepaper für die allgemeinbildenden und berufsbildenden Schulen sowie für die akademische Bildung. Gefordert wird eine flächendeckende Vorbereitung auf die Herausforderungen der heutigen Informationsgesellschaft.

Ausgehend von der zunehmenden Digitalisierung aller Lebensbereiche und dem daraus resultierenden stetigen Wandel des Alltags der Menschen ergeben sich neue Anforderungen, sowohl im beruflichen als auch im privaten Lebensbereich. Die allgegenwärtigen Zugriffsmöglichkeiten auf unerschöpfliche Informationsquellen, die neuen Kommunikationsmöglichkeiten unterschiedlichster Kontexte sowie die neuen Möglichkeiten der gesellschaftlichen Teilhabe und der aktiven Beteiligung an politischen Entscheidungen beeinflussen Kommunikations- und Arbeitsabläufe und ermöglichen durch neue schöpferische Prozesse neue mediale Wirklichkeiten (KMK 2016, 8). Mit Blick auf diese «digitale Revolution» fordert die KMK insbesondere, Bezug nehmend auf den Bildungs- und Erziehungsauftrag der allgemeinbildenden Schulen, ein kompetenzorientiertes und jahrgangsstufenübergreifendes Lernen mit und über digitale Medien sowie die Befähigung zur kritischen Reflexion über selbige (ebd., 11). 
Die KMK formuliert die sechs Kompetenzbereiche «Suchen, Verarbeiten und Aufbewahren», «Kommunizieren und Kooperieren», «Produzieren und Präsentieren», «Schützen und sicher Agieren», «Problemlösen und Handeln» sowie «Analysieren und Reflektieren» für alle Schularten, und damit auch für die Primarschule, wie folgt (die folgenden sechs Abschnitte beziehen sich auf ebd., 15-18):

Der Bereich «Suchen, Verarbeiten und Aufbewahren» thematisiert den Umgang mit Informationen und wird aufgeteilt in die Sektionen «Suchen und Filtern», «Auswerten und Bewerten» und «Speichern und Abrufen». In «Suchen und Filtern» spielen die Klärung und Festlegung verschiedener Arbeits- und Suchinteressen, die Nutzung und Weiterentwicklung von Suchstrategien, das Zurechtfinden in verschiedenen digitalen Umgebungen sowie die Identifikation und Zusammenführung relevanter Quellen eine Rolle. "Speichern und Abrufen» bildet Kompetenzen im Bereich der Analyse, Interpretation und kritischen Bewertung von Daten, Informationen und Informationsquellen ab. Die Sektion «Speichern und Abrufen» behandelt Themen der sicheren Datenspeicherung, -findung und des Abrufs selbiger von verschiedenen Orten, als auch deren Zusammenfassung, Organisation und strukturierten Aufbewahrung.

Der zweite Kompetenzbereich "Kommunizieren und Kooperieren» umfasst die Bereiche «Interagieren», «Teilen», «Zusammenarbeiten», «Umgangsregeln kennen und einhalten (Netiquette)» und «An der Gesellschaft aktiv teilhaben». Während «Interagieren» die Möglichkeiten digitaler Kommunikation und deren ziel- und situationsadäquate Auswahl in den Fokus rückt, stehen in der «Teilen»-Sektion besonders die für die weiterführenden Schulen wichtigen Fähigkeiten im Mittelpunkt: Das Teilen von Dateien, Informationen und Links sowie die Beherrschung einer richtigen Referenzierungspraxis im Sinne einer korrekten Quellenangabe füllen diese Sektion. «Zusammenarbeiten» deckt die Nutzung digitaler Werkzeuge sowohl für die Zusammenarbeit bei der Zusammenführung von Informationen, Daten und Ressourcen, als auch für die gemeinsame Erarbeitung von Dokumenten ab. Die Sektion «Umgangsregeln kennen und einhalten (Netiquette)» fordert die Kenntnis und Anwendung von Verhaltensregeln bei digitaler Interaktion, die Anpassung der Kommunikation an die jeweilige Umgebung, die Kenntnis und Berücksichtigung ethischer Prinzipien bei der Kommunikation sowie die Berücksichtigung der kulturellen Vielfalt in digitalen Umgebungen. Als letzter Teilbereich des Kompetenzbereichs «Kommunizieren und Kooperieren» wird «An der Gesellschaft aktiv teilhaben» aufgeführt, welcher sich mit der Nutzung öffentlicher und privater Dienste, der Weitergabe von Medienerfahrung und Einbringung in kommunikative Prozesse sowie der aktiven Teilhabe an der Gesellschaft als aktiver Bürger beschäftigt.

Im dritten Kompetenzbereich «Produzieren und Präsentieren» werden die praktischen Anwendungsfelder «Entwickeln und Produzieren» und «Weiterverarbeiten und Integrieren» betrachtet. In diesen sollen Inhalte in verschiedenen Formaten mit Hilfe technischer Bearbeitungswerkzeuge unter anderem geplant, gestaltet, bearbeitet 
und veröffentlicht werden sollen, aber auch Informationen, Inhalte und vorhandene digitale Produkte weiterverarbeitet und in bestehendes Wissen integriert werden. Hierbei soll auch die Bedeutung und Berücksichtigung von Urheberrecht, geistigem Eigentum und Persönlichkeitsrechten im Teilbereich «Rechtliche Vorgaben beachten» vermittelt werden.

«Schützen und sicher Agieren» umfasst als eigener Kompetenzbereich die Teilbereiche «Sicher in digitalen Umgebungen agieren», «Persönliche Daten und Privatsphäre schützen», «Gesundheit schützen» und «Natur und Umwelt schützen». In der Sektion «Sicher in digitalen Umgebungen agieren» sollen Risiken und Gefahren in digitalen Umgebungen erkannt, reflektiert und berücksichtigt werden und Strategien zum Schutz entwickelt und angewendet werden. «Persönliche Daten und Privatsphäre schützen» umfasst Massnahmen für Datensicherheit, gegen Datenmissbrauch sowie zur Privatsphäre in digitalen Umgebungen sowie das Treffen und Aktualisieren entsprechender Sicherheitseinstellungen. Ausserdem soll eine Berücksichtigung von Jugendschutz- und Verbraucherschutzmassnahmen erfolgen. Der Teilbereich «Gesundheit schützen» vermittelt Kenntnisse im Bereich der Suchtgefahren sowie zum gesundheitsbewussten, sozial förderlichen und eingliedernden Nutzung digitaler Medien. Die Sektion «Natur und Umwelt schützen» fordert die Berücksichtigung von Umweltauswirkungen digitaler Technologien.

Als fünfter Kompetenzbereich umfasst «Problemlösen und Handeln» die Lernbereiche «Technische Probleme lösen», «Werkzeuge bedarfsgerecht einsetzen», «Eigene Defizite ermitteln und nach Lösungen suchen», «Digitale Werkzeuge und Medien zum Lernen, Arbeiten und Problemlösen nutzen» sowie «Algorithmen erkennen und formulieren». Schwerpunkte im Teilbereich «Technische Probleme lösen» werden hierbei auf die Anforderungen an digitale Umgebungen, die Identifikation technischer Probleme sowie die Bedarfsermittlung und Lösungsfindung gesetzt. Die Anforderungsanalyse, Kenntnis, Anpassung und kreative Anwendung digitaler Werkzeuge zur Problemlösung bildet den Kern des Teilbereichs «Werkzeuge bedarfsgerecht einsetzen». «Eigene Defizite ermitteln und nach Lösungen suchen» formuliert einen selbstreflexiven Kompetenzbereich, bei dem sowohl eigene Defizite in Bezug auf digitale Werkzeuge erkannt und beseitigt werden sollen, als auch eigene, erfolgreiche Strategien zur Problemlösung mit anderen geteilt werden sollen. Das Finden, Bewerten und Nutzen effektiver digitaler Lernmöglichkeiten und die Organisation eines persönlichen Systems vernetzter digitaler Lernressourcen zeichnen den Teilbereich «Digitale Werkzeuge und Meiden zum Lernen, Arbeiten und Problemlösen nutzen» aus. In der Sektion «Algorithmen erkennen und formulieren» werden Kenntnisse zu Funktionsweisen und grundlegenden Prinzipien der digitalen Welt vermittelt, algorithmische Strukturen in genutzten digitalen Tools sollen erkannt und formuliert werden können und strukturierte, algorithmische Sequenzen zur Lösung von Problemen sollen geplant und verwendet werden können. 
Der letzte Kompetenzbereich «Analysieren und Reflektieren» beinhaltet die Bereiche «Medien analysieren und bewerten» und «Medien in der digitalen Welt verstehen und reflektieren». Hierbei soll die Analyse und Bewertung anhand diverser Gestaltungsmittel digitaler Medienangebote und der interessensgeleiteten Setzung, Verbreitung und Dominanz von Themen in digitalen Umgebungen erfolgen, wobei auch Wirkungen von Medien in der digitalen Welt analysiert und konstruktiv reflektiert werden sollen. "Medien in der digitalen Welt verstehen und reflektieren» umfasst die Kenntnis der Vielfalt, Chancen und Risiken der digitalen Medienlandschaft sowie die Bewertung von Vorteilen und Risiken von Geschäftsaktivitäten und Services im Internet. Auch die wirtschaftliche Bedeutung der digitalen Meiden und digitalen Technologien, die Bedeutung digitaler Medien für die politische Meinungsbildung und Entscheidungsfindung sowie die Potenziale der Digitalisierung im Sinne sozialer Integration und sozialer Teilhabe sind hier inkludiert.

Die aufgeführten Kompetenzbereiche beschreiben zum einen die aktuellen Anwendungsgebiete der Digitalen Bildung im deutschen Bildungsraum, zum anderen aber auch das implizit zugrunde gelegte Verständnis der einzelnen Perspektiven und Komponenten Digitaler Bildung durch die Kultusministerkonferenz in Deutschland.

\section{Kritiken zum Strategiepaper «Bildung in der digitalen Welt»}

Die Gesellschaft für Informatik kritisierte bereits in der Entwurfsversion des Strategiepapers in einer Stellungnahme neben Begrifflichkeiten und eingeschränkten Sichtweisen auf den Terminus der «digitalen Welt» besonders die von der KMK formulierten Ziele. Auf Grundlage der Tatsache, dass der digitale Wandel unserer Gesellschaft vor allem durch informatisch qualifizierte Personen gestaltet wird und der Prozess der Digitalisierung massgeblich von technologischen Innovationen geprägt ist, betont Brinda den allgemeinbildenden Charakter des Schulfachs Informatik, die bereits erfolgte Eingliederung in die schulischen Curricula der Bundesländer und die nachgewiesene Erfordernis informatischer Kompetenzen anhand etablierter Bildungsbegriffe (Brinda 2016, 2-3). Aufgrund dieser Gegebenheiten schlussfolgert Brinda:

"die Vermeidung schulorganisatorischer Herausforderungen durch Erweiterung des Pflichtfachkanons wird damit über die Bildungsanforderungen aus einer sich massiv gewandelten und sich stetig und rasant weiter verändernden Lebens- und Arbeitswelt gestellt.» (ebd., 3)

Anzumerken ist an dieser Stelle, dass auch in die 53-seitige finale Version von «Bildung in der digitalen Welt» der Begriff der Informatik keinen Einzug gefunden hat und kein einziges Mal erwähnt wird. Hinzugefügt wurde lediglich, dass notwendige Kompetenzen für ein Leben in einer digitalen Welt über informatische Grundkenntnisse hinausgehen und Anforderungen für eine schulische «Bildung in der digitalen 
Welt» auch in bewährte Konzepte der informatischen Bildung aktualisiert und konkretisiert werden müssten (KMK 2016, 11-12).

Inwiefern informatische Bildung in einer «Bildung in der digitalen Welt» eine Rolle spielt, bleibt somit vorerst unklar. Scheinbar synonym zu Kompetenzen zur Erfüllung des Bildungs- und Erziehungsauftrags in einer «digitalen Welt» wird jedoch, zumindest im Kontext der Lehrerbildung, der Begriff medialer und medienpädagogischer Kompetenzen gebraucht (ebd., 24).

Die Gesellschaft für Medienpädagogik und Kommunikationskultur (GMK) fordert in ihrer Stellungnahme zum Entwurf des Strategiepapiers zunächst eine ternäre Betrachtung der digitalen Welt: eine informationstechnologische/informatische Perspektive, die Perspektive der Mediatisierung und der Entwicklung der digitalen Medien und die Perspektive informatisch-medialer Systeme im Sinne einer Verknüpfung klassischer Medienfunktionen mit Eigenschaften von Informatiksystemen (GMK 2016, 2). Ähnlich zur GI kritisiert auch die GMK das Verhältnis des Papiers zum eigenen Fachbereich und zur KMK-Erklärung «Medienbildung in der Schule» von 2012, ausserdem wird die Bedeutung der bereits etablierten fächerübergreifenden Bildungsleitlinie zur Medienerziehung und informationstechnischen Bildung in der Schule betont (ebd., 2-4).

Letztlich wird besonders die Gefahr einer unzureichenden Bedenkung individueller und sozialer bzw. gesellschaftlicher/kultureller Einflüsse durch den einseitig gesetzten Fokus auf die Digitalisierung befürchtet. Die Einbringung dieser Perspektiven wird sowohl von der GMK als auch von der GI gefordert (GMK 2016, 12; Brinda et al., 11). Auch wird im Sinne eines «Lernen über Medien» die verstärkte Behandlung informatischer Inhalte im Sinne einer (informations-)technologischen Perspektive empfohlen (GMK 2016, 8; Brinda et al., 5).

Abseits etwaiger Differenzen zwischen dem final formulierten Beschluss der KMK und den Anregungen der GI und der GMK sind sich alle Instanzen einig, dass das Lernen mit und über digitale Medien und Werkzeuge möglichst früh beginnen muss, namentlich in der Grundschule (KMK 2016, 11; GMK 2016, 5; Brinda et al., 10). Als Reaktion auf den endgültigen Beschluss bekräftigte dies auch Radon, stellvertretend für Microsoft und den Digitalen Bildungspakt für Deutschland und fordert, «unbedingt auch alle Grundschulen in die Digitalisierungsstrategie mit einzubeziehen» (Radon, zitiert nach Digitaler Bildungspakt 2016b).

Sowohl Vertreter der Informatik als auch der Medienpädagogik fordern schon seit langer Zeit eine Etablierung bereits in der frühkindlichen Bildung (vgl. hierzu u.a. Borowski et al. 2010; Romeike und Reichert 2011; Tulodziecki 1998/2001; Sacher 2003). Fraglich ist allerdings, welche konkreten Inhalte für Schülerinnen und Schüler für einen kritisch-kompetenten Umgang mit Medien und Informationen tatsächlich relevant sind. 
Auch wenn die Informatik in Deutschland bislang lediglich teilweise am Gymnasium als Pflichtfach etabliert ist (auch das allerdings nicht in allen Bundesländern) und der Medienpädagogik eine separate Verankerung als eigenes Fach bislang komplett fehlt, sind für die weitere schulische Bildung der Kinder gleichsam die schulartund fächerübergreifenden Bildungs- und Erziehungsziele relevant, welche in Bayern auch die Medien- und digitale Bildung beinhalten, bei welchen die Schülerinnen und Schüler Kenntnisse und Fähigkeiten zum sachgerechten, selbstbestimmten und verantwortungsvollen Handeln in einer multimedial geprägten Gesellschaft erwerben und durch Analyse und Bewertung von Vorgängen und Gefahren von Medien zu einer bewussten und reflektierten Nutzung selbiger befähigt werden sollen (Staatsinstitut für Schulqualität und Bildungsforschung München 2017).

Unsere Informations-, Medien- und Wissensgesellschaft erfordert somit für einen kompetent-kritischen Umgang mit Medien und Informationen zwangsläufig eine Erweiterung des Fächerkanons um die Komponente der Digitalen Bildung, wobei hierbei die Kritiken der Informatik und Medienpädagogik bei der Konstruktion eines Curriculums bedacht werden sollten. Bevor allerdings eine solche Implementierung in das Curriculum der Primarschule oder die Curricula der weiterführenden Schulen erfolgen kann, gilt es zunächst, sich unabhängig von der konkreten Umsetzung mit den zu vermittelnden Kompetenzen, Inhalten und Perspektiven Digitaler Bildung auseinanderzusetzen und deren Relevanz für den Alltag und die Zukunft der Schülerinnen und Schüler abzuwägen.

Die Lerninhalte sollen in den nächsten Kapiteln aus dem umfangreichen Kompetenzkanon der beiden hauptsächlich beteiligten Fächer Informatik und Medienpädagogik extrahiert werden und deren Perspektiven auf Gemeinsamkeiten untersucht werden. Hierbei soll im Folgenden keine strikte Trennung der beiden Disziplinen erfolgen, sondern ein integrativer Ansatz Anwendung finden.

\section{Perspektive der Medienpädagogik}

Dass die Nutzung von und der Umgang mit Medien Einfluss auf die kindliche Entwicklung und Entfaltung hat, ist unbestritten. Die Mediensozialisationsforschung thematisiert hierbei als eigene Fachdisziplin unter anderem «Medienauswirkungen auf Kinder und Jugendliche, die sich auf den Prozess des Aufwachsens, des Sich-Veränderns und Werdens beziehen» (Sander und Vollbrecht 1987, 29). Baacke, Ferchhoff und Vollbrecht erörtern in diesem Zusammenhang die Sorge,

"ob die noch in ihrer kognitiven, moralischen, emotionalen und physischen Entwicklung stehenden Kinder und Jugendlichen die notwendigen Wahrnehmungskompetenzen und die kognitive und moralische Urteilskraft besitzen, um die Künstlichkeit, Zerrissenheit und Widersprüchlichkeit, Vielschichtigkeit 
und Permanenz von Information durch ständige Medienkonfrontation für sich sinnvoll in einen Zusammenhang zu bringen» (Baacke, Ferchhoff \& Vollbrecht 1997, 20).

Tulodziecki und Six $(2000,361)$ unterteilen die Medienerziehung in vier Aufgabenbereiche:

- Auswählen und Nutzen von Medienangeboten,

- Gestalten und Verbreiten eigener Medienbeiträge,

- Verstehen und Bewerten von Mediengestaltungen sowie

- Erkennen und Aufarbeiten von Medieneinflüssen.

Hierbei beschäftigen sich die einzelnen Bereiche nach Tulodziecki und Möller (1998, 12ff.) mit verschiedenen Aspekten des kompetent-kritischen Umgangs mit Medien:

Der erste Bereich «Auswählen und Nutzen von Medienangeboten» widmet sich der mit der an Bedürfnissen und Interessen orientierten, funktionsabhängigen Auswahl und sinngerechten Nutzung von Medienangeboten unter Abwägung von Handlungsalternativen. Als Nutzungsszenarien für die Schülerinnen und Schüler werden Unterhaltung, Information, Spielen und Lernen angeführt. Sollen die Lernenden zum "Gestalten und Verbreiten eigener Medienbeiträge» angehalten werden, müssen diese lernen, durch entsprechende Kommunikationskompetenz eigene Aussagen medial zu vermitteln, beispielsweise in Bildern/Fotos, Hörbeiträgen und Druckerzeugnissen. Das «Verstehen und Bewerten von Mediengestaltungen» versucht die Kinder zu einer angemessenen Bewertung und einem angemessenen Verständnis von Mediengestaltungen zu befähigen. Hierbei spielen Darstellungsformen, Gestaltungstechniken sowie Gestaltungsabsichten eine Rolle. Im Rahmen des «Erkennens und Aufarbeitens von Medieneinflüssen» soll ein Bewusstsein für den Einfluss der Medien auf sich selbst und andere geschaffen werden, hierbei wird der Fokus auf Gefühle, Vorstellungen und Verhaltensorientierungen gelegt (Tulodziecki und Möller 1998, 12ff.).

Die Medienpädagogik sieht in der Digitalen Bildung somit sowohl die Wahrnehmungs-, Nutzungs- und Konstruktionsprozesse von und mit Medien als Aufgabengebiete, aber auch die kritische Verarbeitung und Bewertung von Medien und deren Einflüssen als Kompetenzbereiche.

\section{Perspektive der Informatik}

2016 wurde in Dagstuhl unter gemeinsamer Arbeit von Medienpädagogen, Informatikern und Vertretern aus der Wirtschaft die Dagstuhl-Erklärung als Stellungnahme zu den Themen Digitale Kompetenzen, Digitalisierung und Digitale Schule verfasst. Im Rahmen der Dagstuhl-Erklärung weisen die Autorinnen und Autoren sowie die Unterzeichnenden (Brinda et al. 2016) der «Bildung in der digitalen vernetzten Welt», kurz: Digitale Bildung, eine essenzielle Rolle für die Allgemeinbildung in einer digital 
geprägten Gesellschaft zu. Kernaufgaben der Allgemeinbildung, darunter «Förderung von Verantwortungsbewusstsein, Urteilsfähigkeit, Kreativität, Selbstbestimmtheit, Partizipation und Befähigung zur Teilnahme am Arbeitsleben» (Brinda et al. 2016, 2) erfordern im Rahmen der Digitalen Bildung eine Verknüpfung von Inhalten und Kompetenzen der Informatik und Medienbildung. Hierbei erfolgt eine trinäre Betrachtung der Phänomene, Gegenstände und Situationen der digitalen vernetzten Welt, durch welche sich die Digitale Bildung in Form von drei Perspektiven widerspiegelt:

Die technologische Perspektive wirft einen kritisch hinterfragenden und bewertenden Blick auf die Funktionsweise der Systeme der digitalen vernetzten Welt. Sie beschäftigt sich insbesondere mit den Wirkprinzipien, Erweiterungs- und Gestaltungsmöglichkeiten dieser Systeme und greift hierbei immer wiederkehrende Konzepte durch die Vermittlung grundlegender Problemlösestrategien und -methoden auf, um die technologischen Grundlagen und das Hintergrundwissen für eine Mitgestaltung der digitalen vernetzten Welt zu schaffen (ebd., 3).

«Die gesellschaftlich-kulturelle Perspektive untersucht die Wechselwirkungen der digitalen vernetzten Welt mit Individuen und der Gesellschaft» (Brinda et al. 2016, 3). Insbesondere werden Fragen nach den Einflüssen digitaler Medien auf Individuen und Gesellschaft, der kritischen Beurteilung von Informationen, dem Entwickeln eigener Standpunkte sowie der Einflussnahme auf gesellschaftliche und technologische Entwicklungen behandelt. Ausgelotet werden ausserdem Möglichkeiten zur Mitgestaltung der digitalen Kultur und Kultivierung durch Gesellschaft und Individuen (ebd.).

Die anwendungsbezogene Perspektive beschäftigt sich mit dem Prozess und der Begründung einer «zielgerichtete(n) Auswahl von Systemen und deren effektive und effiziente Nutzung zur Umsetzung individueller und kooperativer Vorhaben» (ebd., 3). Dies inkludiert die Orientierung in Bezug auf vorhandene Möglichkeiten und Funktionsumfänge sowie die sichere Handhabung diverser gängiger Werkzeuge verschiedener Anwendungsbereiche (ebd.).

\section{Begriffsdiskussion Digitale Bildung}

Die bisher betrachteten Ansätze definieren den Begriff der Digitalen Bildung nicht direkt. Dennoch liegt ihnen jeweils ein implizites Verständnis zugrunde, welches hier kurz zusammengefasst werden soll.

Die KMK setzt einen starken Fokus auf Information und Medien (s.o.): Digitale Bildung umfasst die Suche, Verarbeitung, Speicherung und Kommunikation von Information sowie die Produktion, Präsentation, Analyse und Reflexion von Medienprodukten. In einer sozialen Komponente beinhaltet sie Kommunikation, Problemlösefähigkeit und Handeln im digitalen Raum. Eine Dreiteilung der digitalen Bildung würde somit Informationen, Medien und soziale Interaktion im digitalen Raum umfassen. 
Aus Sicht der Medienpädagogik bilden Wahrnehmungs-, Nutzungs- und Konstruktionsprozesse von und mit Medien sowie die kritische Verarbeitung und Bewertung von Medien den Rahmen der Digitalen Bildung.

Mit der Dagstuhl-Erklärung wird konstatiert, dass eine monodisziplinäre Betrachtung Digitaler Bildung nicht möglich ist. Digitale Bildung beschreibt somit die Betrachtung der Phänomene, Gegenstände und Situationen der digitalen vernetzten Welt aus technologischer, gesellschaftlich-kultureller und anwendungsbezogener Perspektive.

Zusätzlich zu diesem implizit gegebenen Rahmen der Digitalen Bildung sollen im Folgenden explizite Definitionen Digitaler Bildung erörtert werden.

Das «Netzwerk Digitale Bildung», eine Austauschplattform für Akteure aus Schule, Hochschule und Arbeitswelt zum Thema Digitale Bildung, insbesondere für Vertreter aus der Praxis, sieht im Begriff der digitalen Bildung einen vielschichtigen und lebenslangen Lernprozess und definiert diesen wie folgt:

«Digitale Bildung beschreibt, wie sich der gesamte Prozess der Bildung mit dem Einsatz digitaler Medien und auch in Hinblick auf das Bildungsziel grundlegend verändert. Bildung ist ein kontinuierlicher Prozess, der Menschen befähigt, ihr Leben und Lernen in einer digitalisierten Welt aktiv zu gestalten. Dabei geht es nicht mehr um den Erwerb von Faktenwissen - viel bedeutender wird die Kompetenz, sich Wissen selbstorganisiert anzueignen, es anzuwenden und kreative Lösungen für Problemstellungen eigenständig entwickeln zu können» (www.netzwerk-digitale-bildung.de 2016, 2).

Hierbei werden die Elemente «Wissen über und der Umgang mit digitalen Medien», «spezifische Methoden für das Lernen mit digitalen Medien und Inhalten», «Inhalte, die auf das Leben in einer digitalisierten Welt abgestimmt sind» und "eine veränderte Beziehung zwischen Lernenden und Lehrenden» als formgebend für den Prozess der Digitalen Bildung verstanden. Im Zuge dieses Definitionsversuchs wird auch ein kurzer Abriss der historischen Entwicklung Digitaler Bildung gegeben: Nach der Jahrhunderte währenden Tradition von Kreidetafeln und Schulbüchern (seit dem 17. Jhd.) fanden im 20. Jhd. Radios, Film- und Overheadprojektoren sowie Fernsehgeräte zu inhaltlichen Veranschaulichungszwecken Einzug in die Klassenzimmer. Durch die Einrichtung von Sprachlaboren und Computerräumen in den 1980er und 1990er Jahren, später auch durch die Angliederung der Schulen an das Internet, wurden neue Möglichkeiten zur Differenzierung und Individualisierung, als auch neue Formen des kollaborativen Arbeitens ermöglicht. Anfang der 1990er Jahre wurden erste interaktive Whiteboards in Fachräumen und Klassenzimmern installiert, was Schülerinnen und Schülern erlaubte, an einem einzigen Gerät visuell, auditiv und über Berührungssteuerung zu lernen, sowie erarbeitete Inhalte abzuspeichern. Neuere Entwicklungen binden mobile Geräte in Form von Laptops, Tablets und Smartphones mit in den Unterricht ein, was eine noch stärkere Individualisierung der Lernprozesse ermöglicht (www.netzwerk-digitale-bildung.de 2016, 2-3). 
Nach Könnecke umfasst Digitale Bildung «den Erwerb von Wissen und Kompetenzen, welche Menschen dazu befähigen, an einer medialen und technischen Welt teilzuhaben» (Könnecke 2016; www.excitingedu.de/digitale-bildung/)

Mit den Lernprozessen zum Erwerb dieser Kompetenzen beschäftigt sich der «Monitor Digitale Bildung». Hierbei verwenden Schmid et al. eine eher technische Betrachtung:

«Digitales Lernen umfasst alle Lernprozesse, in denen stationäre Computer oder mobile Endgeräte zum Einsatz kommen. Damit ist eine große Bandbreite abgedeckt - von der bloßen Unterstützung des Präsenzlernens durch z. B. digitale Videofilme (YouTube) bis hin zum selbst gesteuerten Lernen innerhalb einer Lernumgebung (z.B. Lernmanagementsystem). Inhaltlich wird das Prinzip «Using ICT (Information and Communication Technology) to learn» betont: also digitale Medien als Lernwerkzeuge, im Gegensatz zu «Learning to use ICT», wo die Handhabung digitaler Medien als Selbstzweck erlernt wird (z. B. Anwendung eines Office-Programms)» (Schmid et al. 2016, 11)

Unterschieden wird hier also zwischen der Unterstützung des klassischen Fachunterrichts durch technische Medien und dem Aneignung von Fertigkeiten zur Bedienung und Nutzung selbiger. Diese sehr radikale Perspektive beschränkt daher digitale Kompetenzen auf eine reine Nutzerperspektive: Wer digitale Medien bedienen kann, ist digital gebildet.

Eine ähnliche, allerdings etwas umfassendere Perspektive nehmen Opiela und Weber (2016) in einem Diskussionspaper zur Digitalen Bildung des «Kompetenzzentrums Öffentliche Informationstechnologie» ein: Digitale Bildung wird in die zwei Kategorien «Medienbildung» und «Digital Mainstreaming» aufgeteilt. Hierbei fokussiert die Medienbildung den «Erwerb von IT-Fähigkeiten und von Kompetenzen für das souveräne Auftreten in der digitalisierten Welt» (Opiela und Weber 2016, 10). Umfasste Bereiche bilden hierbei die Bedienung technischer Medien sowie deren Softwarekomponenten, der verantwortungsvolle Umgang mit sozialen Medien/den eigenen Daten im Netz, ein Bewusstsein für Risiken wie Internetsucht und Cybermobbing sowie Online-Recherche und Datenanalyse. Diesen Fokus auf Digitale Bildung setzt laut Opiela und Weber auch die «Digitale Strategie 2025» des Bundesministeriums für Wirtschaft und Energie (ebd.).

Unter Digital Mainstreaming verstehen Opiela und Weber die «Nutzung und Integration digitaler Medien bei der Wissensvermittlung» (ebd., 10). Mit Hilfe digitaler Lehr- und Lernmittel sollen fachliche Kenntnisse auf neue Weise vermittelt werden. Hierbei werden digitale Medien als integraler und selbstverständlicher Bestandteil des Lernens angesehen - analog zu ihrer Rolle im alltäglichen Leben» (ebd.). Hier werden grosse Chancen bezüglicher individueller Förderung und Wissensvermittlung gesehen. Opiela und Weber weisen auf die komplementäre Beziehung von Medienbildung und Digital Mainstreaming hin. Um die positiven Potenziale der Digitalisierung 
für den Bildungssektor optimal ausschöpfen zu können und etwaige Risiken adressieren und verringern zu können, müssten beide Komponenten in der Bildungsstrategie gleichermassen Berücksichtigung finden (ebd.).

Zusammenfassend lassen sich die Definitionen in verschiedene Kategorien einteilen: Während das «Netzwerk Digitale Bildung» eine wissensbezogene Perspektive einnimmt und Schmid et al. die technische Perspektive im Sinne der Anwendung betrachten, ergeben sich bei Opiela und Weber sowie Könnecke allgemeinere Blickwinkel auf das durch den Erwerb von Kompetenzen veränderte Verhalten des Nutzers in der Gesellschaft.

\section{Ein Verständnis Digitaler Bildung}

Nach Darstellung aktueller Anwendungsgebiete der Digitalen Bildung in der deutschen Bildungspolitik sowie den hierbei geforderten Kompetenzbereichen, sowie den Perspektiven der Medienpädagogik und der Informatik auf die Digitale Bildung soll nun, in Anlehnung an bereits bestehende implizite und explizite Definitionen Digitaler Bildung ein gemeinsame Verständnis der Digitalen Bildung erarbeitet werden. Wie bereits zu Beginn der Arbeit angemerkt, erhebt die Arbeit keinen Anspruch auf Vollständigkeit. Jedoch sollen durch die Heterogenität der Blickwinkel gemeinsame Merkmale herausgearbeitet werden.

Vergleicht man die erarbeiteten Definitionen und Betrachtungsweisen Digitaler Bildung, so kristallisieren sich folgende Komponenten heraus:

- Digitale Bildung umfasst den kritisch-reflektierenden Umgang mit Informationen, Medien und sozialer Interaktion in digitalen Räumen.

- Digitale Bildung thematisiert die Wahrnehmung, Nutzung und Konstruktion von und mit Medien sowie die kritische Verarbeitung und Bewertung von Medien.

- Digitale Bildung betrachtet Phänomene, Gegenstände und Situationen der digitalen vernetzten Welt aus technologischer, gesellschaftlich-kultureller und anwendungsbezogener Perspektive.

- Digitale Bildung befähigt Menschen zur aktiven Lebensgestaltung und zum adäquaten Umgang mit Medien, Informationen und Phänomenen in einer digitalisierten Welt (bzw. bei Könnecke eine «mediale und technische Welt»).

- Digitale Bildung unterstützt klassische Lernprozesse durch den Einsatz und die Nutzung bei der Wissensvermittlung.

- Digitale Bildung beinhaltet die Handhabung digitaler Medien zum Selbstzweck sowie für ein souveränes Auftreten in der digitalisierten Welt, insbesondere auch deren Auswahl und Begründung.

- Digitale Bildung inkludiert den Erwerb von Kenntnissen bezüglich der Funktionsweisen, Wirkprinzipien und technologischen Grundlagen digitaler Systeme sowie hierbei Anwendung findende wiederkehrende Konzepte und Problemlösestrategien. 
- Digitale Bildung betrachtet Wechselwirkungen zwischen Individuen und der Gesellschaft mit der digitalen vernetzten Welt und bildet Kompetenzen zur Informationsbeurteilung sowie zur Entwicklung eigener Standpunkte aus.

Da es sich bei der Unterstützung klassischer Lernprozesse um eine konkrete Anwendung auf Prozesse zum Wissenserwerb handelt, lassen sich diese beiden Punkte zusammenfassen. Um der Integration von Problemlösestrategien und Wirkprinzipien in die technologische Perspektive Rechnung zu tragen wird diese zu einer informatischen Perspektive erweitert. Medien, Informationen und Interaktionen in digitalen Räumen (diese kann unter dem bereits bestehenden weiteren Begriff der Phänomene gefasst werden) bilden den Kern der Digitalen Bildung, welcher aus verschiedenen Perspektiven betrachtet wird.

Konkludierend lässt sich eine erweiterte Form des Dagstuhl-Dreiecks darstellen (Abb. 1):

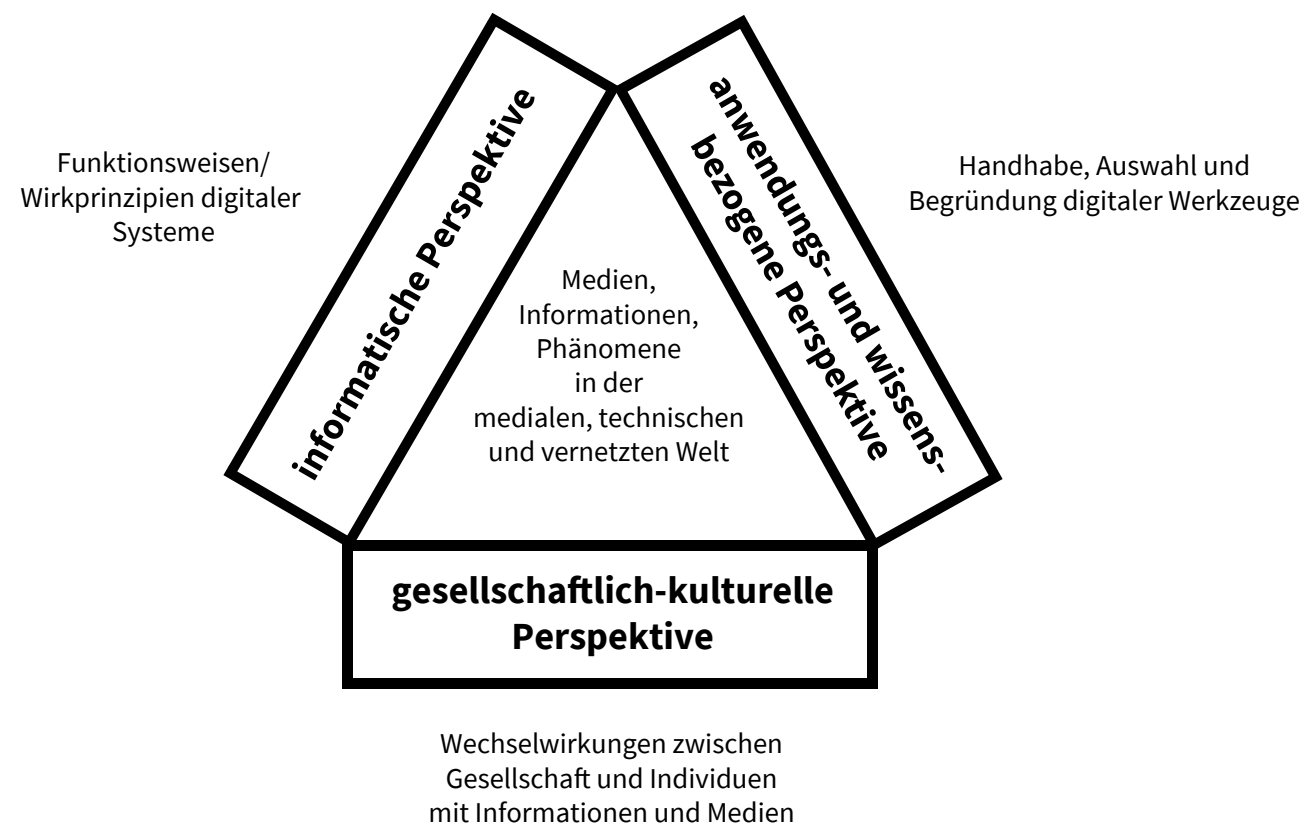

Abb. 1.: Erweitertes Dagstuhl-Dreieck (eigener Entwurf).

Digitale Bildung vermittelt Kompetenzen zur proaktiven und souveränen Lebensgestaltung von Individuen im Umgang mit Medien, Informationen und Phänomenen in der medialen, technischen und vernetzten Welt. Digitale Bildung setzt sich zusammen aus den komplementären Perspektiven der technologischen Funktionsweisen und Wirkprinzipien digitaler Systeme, der Betrachtung reziproker Wechselwirkungen zwischen postfaktischer Gesellschaft und dem Individuum als Prosumenten mit Informationen und Medien sowie der anwendungsbezogenen und wissensbezogenen 
Handhabe, Auswahl und Begründung digitaler Werkzeuge zur individuellen Zielerreichung sowie zur Unterstützung individueller Lernprozesse.

Betrachtet man diese Definition aus dem Fokus «schulische Bildung» mit der Prämisse einer medialisierten Welterschliessung, so müssten im Sinne der Digitalen Bildung die Inhalte eines jeden Fachs, sofern sie durch eine digitale Repräsentation wahrgenommen werden, aus den dargestellten Perspektiven betrachtet werden. Dies integriert sowohl die Reflexion von medial übermittelten Informationen (die Inhalte der Fächer), als auch die Reflexion über die Medien, welche diese vermitteln. Hierfür ist es insbesondere notwendig, das klassische Verständnis der Medienkompetenz aufzubrechen und diese in Verbindung mit informatischen sowie interdisziplinären sozialwissenschaftlichen Kompetenzen zur Digitalen Bildung im Sinne einer Befähigung zur kritischen Informations- und Medienkompetenz in der Informations-, Medien- und Wissensgesellschaft zu erweitern.

Die erforderlichen Fähigkeiten, welche durch die Digitale Bildung also vermittelt werden sollen, gehen über den klassischen Kompetenzbegriff hinaus: Die kritischereflektierende Betrachtung von Informationen, Medien und Phänomenen gleicht einer Fähigkeit des «Lesens» digitaler Inhalte. Es kann also die Rede vom Erfordernis einer «Information, Media and Digital Literacy» sein, welche Einzug in schulische Bildungsdiskurse erhalten muss, wenn Digitale Bildung nicht mehr monodisziplinär, sondern auf inter- und transdisziplinären Wegen betrachtet werden soll. In diesem Kontext ist auch die Rede von einer allgemeinen Digital Literacy mit den Bestandteilen Information Literacy, Media Literacy und Information and Communications Technology Literacy als Schlüsselkompetenzen des 21. Jahrhunderts, welche über gesellschaftlichen und beruflichen Erfolg entscheiden (Trilling und Fadel 2009). Auch von «Metakompetenzen der Wissensgesellschaft» (vgl. Lloyd 2003, 91) ist die Rede. Die Digitale Bildung als grundsätzliche Befähigung einer Teilhabe an der Gesellschaft ist somit allgemeinbildend und muss, bevor sie Einzug in die Lehrpläne findet, zunächst an den Hochschulen vermittelt werden, um qualifizierte Lehrpersonen hervorzubringen, welche diese Inhalte auch unterrichten können. Dies erfordert ein grundsätzliches Überdenken der vorhandenen Modulkataloge der Lehramtsstudiengänge. Auch hier wäre ein interdisziplinärer Ansatz anzudenken. 


\section{Literatur}

Baacke, Dieter, Wilfried Ferchhoff, und Ralf Vollbrecht. 1997. «Kinder und Jugendliche in medialen Welten und Netzen. Prozesse der Mediensozialisation». In Handbuch Medien: Computerspiele, herausgegeben von Jürgen Fritz, und Wolfgang Fehr, 31-57. Bonn: Bundeszentrale für politische Bildung. Bonn.

Borowski, Christian, Ira Diethelm, und Ana-Maria Mearoş. 2010. «Informatische Bildung im Sachunterricht der Grundschule: Theoretische Überlegungen zur Begründung». www.widerstreit-sachunterricht.de, Nr. 15, Oktober 2010. http://www.widerstreit-sachunterricht.de/ ebenel/superworte/infor/BorDieMe.pdf.

Brinda, Torsten. 2016. Stellungnahme zum KMK-Strategiepaper «Bildung in der digitalen Welt». https://fb-iad.gi.de/fileadmin/stellungnahmen/gi-fbiad-stellungnahme-kmk-strategiedigitale-bildung.pdf.

Brinda, Torsten, Ira Diethelm, Rainer Gemulla, Ralf Romeike, Johannes Schöning, Carsten Schulte, und et al. 2016. "Dagstuhl-Erklärung: Bildung in der digitalen vernetzten Welt». Herausgegeben von Gesellschaft für Informatik e.V. https://gi.de/fileadmin/GI/Hauptseite/ Themen/Dagstuhl-Erkla__rung_2016-03-23.pdf.

Digitaler Bildungspakt, Hrsg. 2016a. «Die richtige Bildung für die digitale Welt. Politische Handlungsempfehlungen und Expertenstatements». http://digitaler-bildungspakt.de/wpcontent/uploads/2016/11/Kompendium-Die-richtige-Bildung-fu\%CC\%88r-die-digitaleWelt.pdf.

Digitaler Bildungspakt, Hrsg. 2016b. «Reaktionen auf die Strategie der Kultusminister für Bildung in der digitalen Welt». http://digitaler-bildungspakt.de/2016/12/12/reaktionen-aufdie-strategie-der-kultusminister-fuer-bildung-in-der-digitalen-welt/.

Gesellschaft für Medienpädagogik und Kommunikationskultur. 2016. "Stellungnahme der Gesellschaft für Medienpädagogik und Kommunikationskultur (GMK) zum Strategie-Papier der KMK «Bildung in der digitalen Welt». 27. April. http://www.gmk-net.de/fileadmin/pdf/ GMK-Stellungnahme_zum_KMK-Strategie-Entwurf.pdf.

i-dbnd - Initiative DIGITALE BILDUNG NEU DENKEN. 2017. DIGITALE BILDUNG NEU DENKEN. http://i-dbnd.de/digitale-bildung-neu-denken/.

Könnecke, Tobias. 2016. «excitingedu - Digitale Bildung». http://www.excitingedu.de/digitalebildung/.

KMK - Kultusministerkonferenz. 2016. «Bildung in der digitalen Welt», Beschluss vom 8.12.2016. https://www.kmk.org/fileadmin/Dateien/pdf/PresseUndAktuelles/2016/Bildung_digitale_Welt_Webversion.pdf.

Lloyd, Annemaree. 2003. «Information Literacy: The Meta-Competency of the Knowledge Economy? An Exploratory Paper». Journal of Librarianship and Information Science 35 (2): 87 92. https://doi.org/10.1177/0961000603352003.

Opiela, Nicole, und Mike Weber. 2016. «Digitale Bildung - Ein Diskussionspapier». In Kompetenzzentrum Öffentliche IT, herausgegeben von Fraunhofer-Institut für Offene Kommunikationssysteme FOKUS. Berlin. https://www.oeffentliche-it.de/documents/10181/14412/ Digitale+Bildung+-+Ein+Diskussionspapier. 
Romeike, Ralf, und Dominik Reichert. 2011. «PicoCrickets als Zugang zur Informatik in der Grundschule». In Informatik in Bildung und Beruf (GI-Fachtagung «Informatik und Schule 2011»), herausgegeben von Marco Thomas. 177-186. Münster.

Sacher, Werner. 2003. Medienerziehung konkret: Konzepte und Praxisbeispiele für die Grundschule (Studientexte zur Grundschulpädagogik und -didaktik). Bad Heilbronn: Klinkhard Verlag.

Sander, Uwe, und Ralf Vollbrecht. 1987. Kinder und Jugendliche im Medienzeitalter. Annahmen, Daten und Ergebnisse der Forschung. Leske + Budrich. Opladen.

Schmid, Ulrich, Lutz Goertz, und Julia Behrens. 2016. Monitor Digitale Bildung. Berufliche Ausbildung im digitalen Zeitalter. Gütersloh: Bertelsmann Stiftung. https://www.bertelsmannstiftung.de/fileadmin/files/BSt/Publikationen/GrauePublikationen/Studie_Monitor-Digitale-Bildung_Berufliche-Ausbildung-im-digitalen-Zeitalter_IFT_2016.pdf.

Staatsinstitut für Schulqualität und Bildungsforschung München. LehrplanPLUS Bayern. https://www.lehrplanplus.bayern.de/uebergreifende-ziele.

Trilling, Bernie, und Charles Fadel. 2009. 21st Century Skills: Learning for Life in Our Times. San Francisco: John Wiley \& Sons.

Walden, Thomas. 2016. Trickster, Kreativität und Medienkompetenz. München: Kopaed.

Tulodziecki, Gerhard. 1998. «Entwicklung von Medienkompetenz als Erziehungs- und Bildungsaufgabe». Pädagogische Rundschau 52 (6), 693-709.

Tulodziecki, Gerhard, und Ulrike Six. 2000. Medienerziehung in der Grundschule. Grundlagen, empirische Befunde und Empfehlungen zur Situation in Schule und Lehrerbildung. Verlag für Sozialwissenschaften | Springer Fachmedien Wiesbaden GmbH. Wiesbaden.

www.netzwerk-digitale-bildung.de. 2016. «Zwischen analog und digital. Lernen und Lehren an Schulen und Hochschulen». http://www.netzwerk-digitale-bildung.de/wp-content/uploads/NDB_Whitepaper_Zwischen_analog_und_digital_12Seiten.pdf. 